\title{
Successful retrieval of fine-needle aspiration biopsy material from previously stained smears for immunocytochemistry: a novel technique applied to three soft tissue tumors
}

\author{
Min En Nga ${ }^{1}$, Gaik-Lan Lim $^{2}$, Nilsson Barbro ${ }^{2}$ and Norman HL Chan ${ }^{3}$ \\ ${ }^{1}$ Department of Pathology, National University of Singapore, Singapore; ${ }^{2}$ Department of Pathology, National \\ University Hospital, Singapore and ${ }^{3}$ Tawam Hospital, Al Ain, Emirate of Abu Dhabi, United Arab Emirates
}

\begin{abstract}
Fine-needle aspiration biopsies of soft tissue tumors are often not fully diagnostic on conventional Papanicolaou or Giemsa stains. Often, more useful information can be gleaned from preparing cell blocks, which are amenable to immunocytochemistry. However, sufficiently diagnostic cell block material is not always forthcoming. We subvent this problem by employing a new 'cytoscrape' technique to create cell blocks from previously Papanicolaou-stained smear material in two cases of mesenchymal tumors where smear diagnoses were limited to 'sarcoma, not otherwise specified'. After thorough text and photographic documentation of the smear results, the slides were decolorized and tumor cells were removed for processing as for routine preparation of cell blocks. Sections cut were then submitted for immunocytochemical profiling. This resulted in definitive diagnoses in both cases, averting the need for more invasive diagnostic procedures. We applied the same technique to a third case of a lung nodule, in which the smear was inconclusive and the original cell block nondiagnostic. The subsequent immunocytochemical testing enabled a definitive diagnosis to be made. This novel technique is described here; its value and applicability are discussed in the context of fine-needle aspiration biopsies of soft tissue tumors.

Modern Pathology (2005) 18, 728-732, advance online publication, 3 December 2004; doi:10.1038/modpathol.3800356
\end{abstract}

Keywords: cell block; fine-needle aspiration biopsy/cytology; immunocytochemistry; soft tissue tumors

Conventional practice in cytopathology does not often explore the possible advantages of reprocessing material from Papanicolaou (Pap)- and Giemsastained slides. In particular, the preparation of cell blocks has not been described in this context. We describe a novel method of preparing cell blocks using material harvested from previously stained cytological smears. This method was applied in three cases of soft tissue tumors, which allowed valuable additional information by way of immunocytochemistry. Definitive diagnoses were obtained in two cases by a combination of cytomorphological and immunohistochemical features; in the third case a retrospective look at the cell block material yielded enough information to strongly suggest the tissue diagnosis.

Correspondence: Dr ME Nga, MBBS, Department of Pathology, National University of Singapore, 5 Lower Kent Ridge Road, Singapore 119074, Singapore.

E-mail: patngame@nus.edu.sg

Received 6 October 2004; revised and accepted 3 November 2004; published online 3 December 2004

\section{Methods}

Three cytology cases seen in the Department of Pathology of the National University Hospital of Singapore were selected. Cytologic material consisted of smears from fine-needle aspiration biopsies of the liver (case 1), the inferior vena cava (case 2) and the peripheral lung (case 3). Aside from case 3 (referred to our center as a case for second opinion), smears were prepared and stained in the usual manner, yielding two Giemsa- and two Pap-stained slides for each case. No material was available for cell block in two of the cases; however, a cell block was prepared for case 3 by conventional means, using material in the hub of the fine-needle aspiration biopsy needle fixed in Bouin's solution. Smears from all three cases were examined, described and photographed. One Pap-stained smear was chosen from each case, based on the presence of large, cohesive cellular clusters that were visible to the naked eye. For each case, the coverslip was removed by immersion in xylene, and the smear was decolorized by rinsing for $10 \mathrm{~s}$ in $0.5 \%$ acid alcohol 
solution. The cohesive cell clusters were gently scraped off the surface of the slide using a size 22 surgical blade, and transferred into cassettes lined with filter paper. The tissue was then processed as for routine paraffin embedding in the usual manner for cell blocking. Serial $4 \mu \mathrm{m}$ thick sections were cut and stained with hematoxylin and eosin $(\mathrm{H}+\mathrm{E})$, as well as the relevant immunocytochemical stains to aid diagnosis. Immunocytochemical stains were carried out by the avidin-biotin method, using microwave antigen retrieval.

\section{Case description}

\section{Case 1}

A 30-year-old Indonesian Chinese male presented with abdominal pain for one and a half years. Computerized tomogropahy (CT) scans showed multiple liver and spleen lesions. Fine-needle aspiration cytology of the liver was performed in another center, the reported diagnosis being 'malignant spindle cell lesion suggestive of a sarcoma'. The slides were sent to our facility for second opinion. A total of 14 Pap, Giemsa or H+E-stained slides were received. These showed malignant cohesive cells with fusiform nuclei (Figure 1) and scant cytoplasm admixed with benign hepatocytes within a hemorrhagic background. As detailed above, tissue scrapings from one of the slides were made and a cell block prepared (Figure 2a). Immunocytochemical stains showed the malignant spindle cells to be reactive for CD31 (Figure 2b), factor VIII and smooth muscle actin. They were negative for S100, AE 1/3 and CD117. With the aid of the immunocytochemical profile and the corroborative cytomorphological picture, a diagnosis of 'sarcoma, compatible with angiosarcoma' was made. The patient later underwent a splenectomy; which

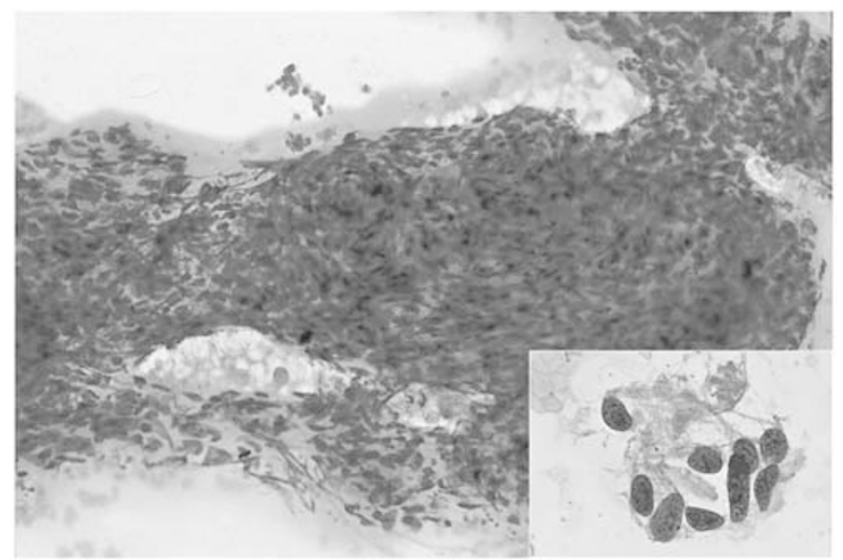

Figure 1 Case 1-medium and high power views of the liver fineneedle aspiration biopsy showing cohesive sheets of overlapping spindled cells with fusiform, pleomorphic nuclei $(\mathrm{H}+\mathrm{E}, \times 200$; inset $\times 600$ ). confirmed the diagnosis of angiosarcoma on histology (Figure 2c).

\section{Case 2}

A 62-year-old Chinese female presented with an inferior vena caval tumor clinically thought to be a leiomyosarcoma. The tumor originated from the renal vein, grew into the inferior vena cava and extended superiorly to the right atrium. A fineneedle aspiration biopsy was performed from the inferior vena caval tumor, Pap and Giemsa preparations were made in the usual manner; there was insufficient material to prepare a cell block. The smears was showed cohesive sheets of malignant spindle cells (Figure 3), consistent with that of a mesenchymal tumor. Scrapings from the largest cluster were retrieved for cell block preparation as outlined above. Subsequent sections underwent $\mathrm{H}+\mathrm{E}$ staining and immunocytochemical staining for smooth muscle actin, desmin and CD117. The immunophenotype matched that of a leiomyosarcoma (smooth muscle actin and desmin strongly positive, CD117 negative). The definitive cytological diagnosis of a vascular leiomyosarcoma was made. The patient did not undergo any additional invasive diagnostic procedures (Figure 4).

\section{Case 3}

A 63-year-old Malay male presented with a peripheral left upper lobe lung nodule. CT scan showed a $2 \times 2 \mathrm{~cm}$ nodule. Previous fine-needle aspiration biopsy in another center suggested the diagnosis of a sclerosing hemangioma (type II pneumocytoma). These slides were not available for review. The procedure was repeated in our facility and yielded a cellular aspirate composed of elongated cells admixed with occasional columnar epithelial cells. The cytological features on Pap and Giemsa preparations were not specific; however, the apparently biphasic nature of the smear raised the possibility of a sclerosing hemangioma or a pulmonary hamartoma, and an excision biopsy was recommended. A cell block was prepared in the conventional manner, using remnant tissue from the needle hub; however, this yielded no lesional tissue. The patient underwent resection of the nodule. Histology showed a pleural-based localized mass composed of interlacing bundles of spindle cells with scattered normal mitotic figures. The nodule showed variable cellularity with a collagenous background. Bronchiolar structures were present at the parenchymal edge of the tumor, with islands of entrapped lung parenchyma near the tumor edge. Immunohistochemical profile was as follows: CD34 and vimentin strongly and diffusely positive; other markers negative (S100, MNF116 and synaptophysin). A diagnosis of solitary fibrous tumor was made. The previously reported cytology smears were revisited and a 

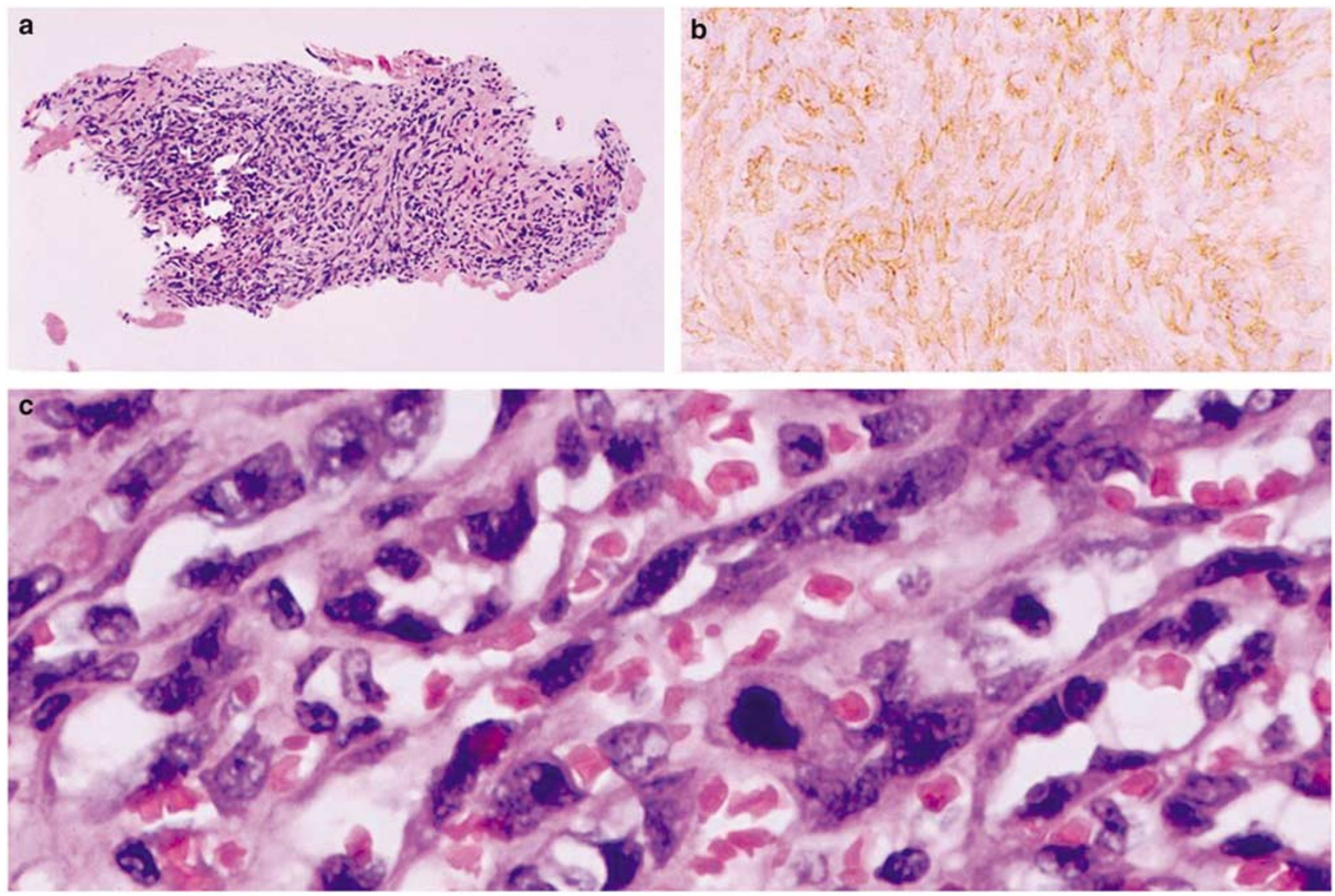

Figure 2 Case 1-(a) section taken of post-cytoscrape cell block $(\mathrm{H}+\mathrm{E}, \times 100)$; (b) strong reactivity for CD31(immunoperoxidase, $\times 400)$; (c) histology of the spleen lesion showing malignant cells forming vascular lumina $(\mathrm{H}+\mathrm{E}, \times 600)$.

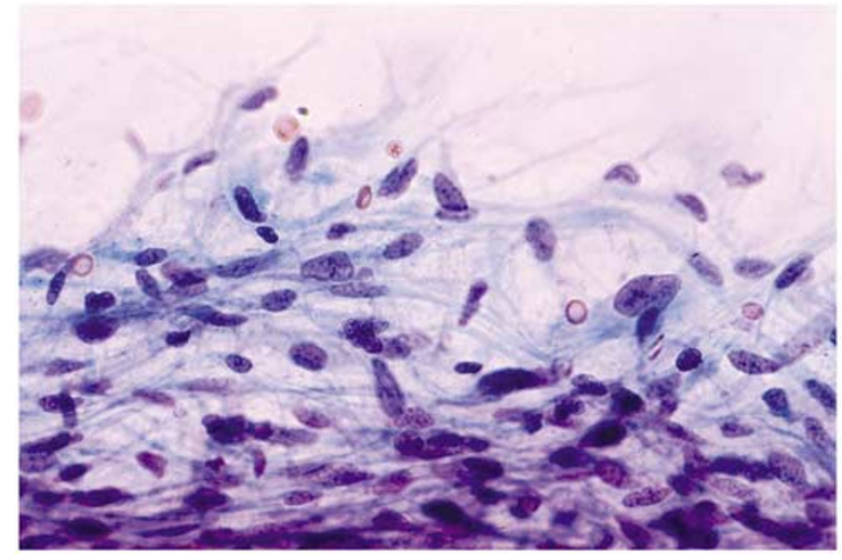

Figure 3 Case 2-smear of inferior vena caval tumor showing pleomorphic, cohesive tumor cells with plump to spindled, overlapping nuclei (Pap stain, $\times 600)$.

suitably cellular slide was chosen. The same 'cytoscrape' technique was applied and the tissue processed as a cell block. $\mathrm{H}+\mathrm{E}$ stains and a panel of immunocytochemical tests were performed. These showed patchy positivity for desmin and strong diffuse positivity for vimentin and CD34. Antibodies to S100, synaptophysin and cytokeratins were negative. Thus, on retrospect, immunocytochemistry of the cell block material could have provided valuable information contributing to the final diagnosis.

\section{Discussion}

To this day, the cytologic diagnosis of soft tissue tumors remains a considerable challenge. The main difficulties lie in the histological subtyping of tumors and the differential diagnosis of sarcomatoid epithelial tumors. This difficulty is further compounded by the fact that the cohesive mesenchymal nature of the tumor cells sometimes leads to scant cytologic material, thus limiting material available for smears and especially cell block preparation. It is, however, this precise quality of these tumors that enables the cells to be scraped off the slide, as they form cohesive clusters that withstand the mechanical stresses of being removed from the smear and embedded in paraffin blocks. Furthermore, these cell clusters are visible to the naked eye and hence amenable to physical manipulation. The great advantage in obtaining cell blocks is clearly manifest in these three cases, where cytologic diagnoses were significantly limited on Pap and Giemsa stains 
a

a

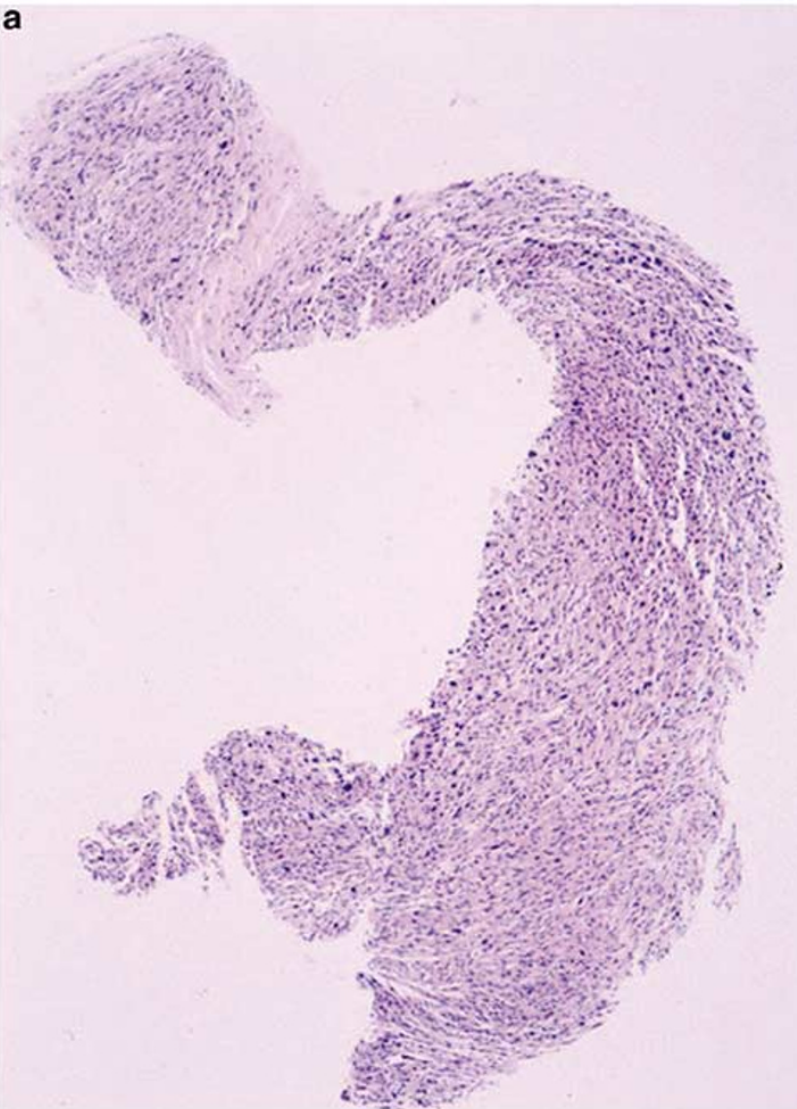

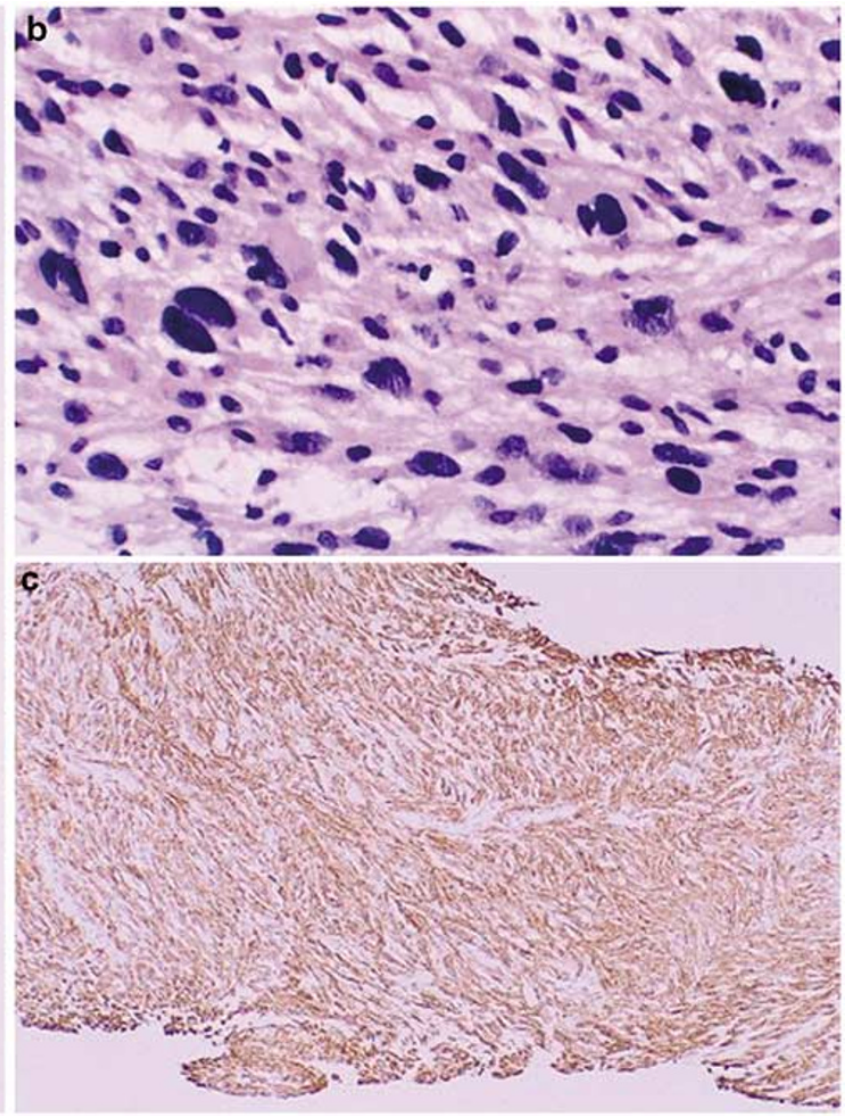

Figure 4 Case 2-sections taken from the cell block after cytoscrape. (a) Medium power section showing cohesive pleomorphic cells with eosinophilic cytoplasm $(\mathrm{H}+\mathrm{E}, \times 100)$; (b) high power view showing marked muclear pleomorphism and hyperchromasia, and relatively good preservation of cell morphology $(\mathrm{H}+\mathrm{E}, \times 400)$; (c) strong positivity for desmin (immunoperoxidase, $\times 200)$.

alone. With the aid of immunocytochemical stains, the definitive diagnoses could be obtained without difficulty. The clinical value of this method is demonstrated especially clearly in case 2-where a definitive diagnosis of leiomyosarcoma of the inferior vena cava meant that no further invasive diagnostic tests needed to be carried out in this anatomically high-risk site.

Recent reviews on the usefulness of fine-needle aspiration cytology in the diagnosis of soft tissue tumors have yielded diagnostic specificities ranging from 21 to $97 \%,{ }^{1-3}$ including the use of ancillary techniques such as cell blocks for immunocytochemistry. However, the intrinsic limitations of this diagnostic method result in a general understanding that grading and histological subtyping of tumors is not always possible. Hence, additional information in the form of special histochemical stains, immunophenotype and proliferation markers (Ki-67, PCNA) is valuable both diagnostically and prognostically, and is now made possible with the advent of cell block technology. One review ${ }^{1}$ of fine-needle aspiration biopsies of soft tissue tumors of 43 patients showed that the correct specific diagnosis was given in only $21 \%$ of cases, including the cases that were deemed to be inadequate. However, a positive diagnosis which led to appropriate diagnostic surgical biopsy was obtained in $88 \%$ of cases. Ancillary tests including the use immunocytochemistry in cell block material were not found to be significantly contributory. A possible reason for this could be that conventionally prepared cell block preparations rely on residual material within needles or syringes. The contents of such material are highly unpredictable, and in some instances yield only hemolysed blood or acellular proteinaceous material. Wakely and Kneisl ${ }^{2}$ found that only 21 of 39 cell blocks contained cellular material, of which only 12 yielded sufficient cytological material for immunophenotyping or special histochemical stains.

In the cell block technique that we describe, the material is 'prescreened' based on the previous cytological evaluation of the smears. This largely eliminates the problem of nondiagnostic cell block material, even if a conventional cell block had been initially prepared for the case.

Kilpatrick et $a l^{4}$ found that, in 145 fine-needle aspiration biopsy specimens from 140 patients, subtyping of adult pleomorphic soft tissue tumors was generally not possible. Although this did not influence the ultimate therapy in the majority of 
patients, we feel that the ability to subtype tumors is clinically relevant in many situations. One such instance involves fine-needle aspiration biopsies of tumors where the differential diagnosis includes a metastatic tumor or a second primary. Also, sarcomatoid carcinomas may be a potential pitfall in the evaluation of spindle cell tumors, where immunocytochemistry is of obvious value. Similarly, epithelioid sarcomas may also pose a problem in the differential diagnosis of true epithelial carcinomas.

The immunocytochemical staining of previously stained cytologic preparations has been studied by Abendroth and Dabbs, ${ }^{5}$ who compared the results of immunoperoxidase staining on unstained smears and previously Pap-stained smears. The latter were divided into two groups: those that were further decolorized in acid alcohol and those that were not decolorized. They found that a spectrum of antibodies (including AE1/3, desmin and HHF35) showed comparable staining in both groups when compared to initially unstained smears. Both the decolorized and nondecolorized group showed good results, that is, $84-87 \%$ were either similar in quality or superior to unstained preparations, in terms of staining intensity and distribution, cell preservation and background staining. However, these are studies that are based on smear material only; they are not in the context of cell block preparation.

Baloch et $a l^{6}$ described a method of cell block preparation using a portion of the Pap-stained Millipore filters from previously concentrated cytology specimens (including pleural effusions and fine-needle aspiration biopsies of solid tumors). The filter was removed from the slide, divided and a portion processed for paraffin embedding in the usual manner. Subsequent sections were taken for immunocytochemistry. There was excellent preservation of cytomorphology with specific patterns of antibody staining using AE1/3, LCA and HMB45. Similarly, Rowe et $a 7^{7}$ have described an effective method of cell block preparation using ThinPrep ${ }^{\mathrm{TM}}$ fluid-based technology in a case of an abnormal gynecological Pap smear. They obtained cell block material from the residual ThinPrep ${ }^{\mathrm{TM}}$ Pap test fluid using a thrombin-based technique. Immunocytochemistry, however, was not applied in this case. The additional cell block $\mathrm{H}+\mathrm{E}$ cytohistologic morphology was sufficient for accurate diagnosis.

These methods of retrieval of cell block material are based on residual material in fluid or filter preparations; however, the conversion of previously stained and read material from cytologic smears to cell block material has not been described prior to this. This is an effective, specific method that allows the cytopathologist to select optimal material for reprocessing in a manner that will yield much additional clinically relevant information. This cytoscrape method is simple and cost-effective, and provided that attention is paid to obtaining good pictorial and written documentation of the previous smears, is a useful adjunct to the cytopathological definitive diagnosis of soft tissue tumors, as is illustrated clearly in the context of these three cases.

\section{Acknowledgements}

We express our thanks to $\mathrm{Mr}$ Ow CK for the immunocytochemical stains and Mr TC Tan for the photography.

\section{References}

1 Costa MJ, Campman SC, Davis RL, et al. Fine-needle aspiration cytology of sarcoma: retrospective review of diagnostic utility and specificity. Diagn Cytopathol 1996;15:23-32.

2 Wakely Jr PE, Kneisl JS. Soft tissue aspiration cytopathology-diagnostic accuracy and limitations. Cancer Cytopathol 2000;90:292-298.

3 Gonzalez-Campora R. Fine needle aspiration cytology of soft tissue tumours. Acta Cytol 2000;44:337-343.

4 Kilpatrick SE, Cappellari JO, Bos GD, et al. Is fineneedle aspiration biopsy a practical alternative to open biopsy for the primary diagnosis of sarcoma? Experience with 140 patients. Am J Clin Pathol 2001;115: 56-68.

5 Abendroth CA, Dabbs DJ. Immunocytochemical staining and unstained versus previously stained cytologic preparations. Acta Cytol 1995;39:379-386.

6 Baloch ZW, Lee A, Cobbs C, et al. Millipore filter cell block preparation: an alternative to cell block in nongynaecologic specimens of limited cellularity. Diagn Cytopathol 1999;20:389-392.

7 Rowe LR, Marshall CJ, Bentz JS. Call block preparation as an adjunctive diagnostic technique in ThinPrep monolayer preparations: a case report. Diagn Cytopathol 2001;24:142-144. 